\title{
Molecular Genetic Diagnosis of the Inherited Bleeding Disorders: Are We Close to the Perfect Test?
}

\author{
Hara Prasad Pati ${ }^{1} \cdot$ Prashant Sharma $^{2}$
}

Published online: 6 October 2016

(C) Indian Society of Haematology \& Transfusion Medicine 2016

This issue includes a list of reviewers who have evaluated manuscripts submitted to the journal over the past year. This will now be an annual feature and is the journal's acknowledgement of our expert reviewers' effort and time.

In this issue, Khaniani et al. [1] from Tabriz in Iran describe their experiences with the genetic diagnosis of haemophilia $\mathrm{A}$ in patients and female carriers. Using an array of molecular techniques including inverse-shifting PCR, long-range PCR, eight multiplex PCRs and Sanger sequencing, the authors achieved a mutation detection rate of $48 \%$, i.e. in 24 out of 50 haemophilia A patients. The mutations detected were not unlike those reported from India, although yields have been higher in Indian studies [2]: 22 of the Iranian cases had the intron 22 inversion, while one each had intron 1 inversion and a point mutation in exon 14 . The study highlights the challenges and limitations of conventional testing strategies for the diagnosis of inherited bleeding disorders and the geographical variability.

Conventional strategies rely on step-wise performance of tests of increasing complexity. While this scientifically valid approach saves costs, it causes delays affecting not just the patients but also in prenatal diagnosis. Individual tests too have specific limitations. Hemostatic screening tests (PT, aPTT and TT) are non-specific and insensitive to mild factor deficiencies [3]. Tests of intermediate complexity like factor assays, platelet aggregometry and flow cytometry are dependent on pre-analytical variables [4]. More definitive tests like multimer-analysis, platelet

Hara Prasad Pati

harappati@yahoo.co.in

1 New Delhi, India

2 Chandigarh, India nucleotide release and vWF-collagen/FVIII binding assays are technically complex with limited availability in only selective referral centers.

Cheap and rapid molecular diagnostics, if available, would therefore be extremely valuable in bleeding disorders. However, as the Khaniani paper [1] illustrates, the heterogeneity of molecular lesions in even the commoner diseases like haemophilia A and B, von Willebrand disease and the commoner platelet function defects make it technically complex to use them as first-line diagnostic tests. Current applications of molecular testing in these disorders are therefore largely restricted to carrier detection and antenatal diagnosis [5]. Less commonly, molecular tools may be applied for the distinction of phenocopies (i.e. type $2 \mathrm{vWD}$ from platelet-type $\mathrm{vWD}$, or moderate haemophilia A from type $2 \mathrm{~N} v W D$ ) or sometimes to obtain clinically relevant genetic information, like haemophilia mutationbased inhibitor risk profiling [2] or to confirm $F 9$ promoter mutations causing haemophilia B Leyden [5] for prognostication.

Sanger sequencing is now a convenient diagnostic tool for many monogenic disorders. Bi-directional sequencing of the entire coding region, intron/exon junctions (i.e. splice sites), and proximal promoter elements of small genes like $F 9$ ( $34 \mathrm{~kb}$ in size with a $2.4 \mathrm{~kb}$ coding region) is diagnostic in $97 \%$ cases of haemophilia B [6]. F8 and $v W F$ genes however, are much larger in size (186 and $178 \mathrm{~kb}$, respectively), have numerous exons (27 and 52, respectively) that are separated by long intronic sequences, large coding sequences ( $\sim 9 \mathrm{~kb}$ in both) and frequently display alternative splicing making it extremely difficult to evaluate them by Sanger sequencing [5-7]. An alternative approach is to first use a screening technique like the conformation-sensitive gel electrophoresis (CSGE) or multiplex ligation-dependent probe amplification (MLPA) 
or array $\mathrm{CGH}$ followed by sequencing of only the implicated exons [6, 8]. However, the additional techniques obviously increase effort, time and cost.

Later generation sequencing techniques have overcome many of the shortcomings of the conventional molecular assays. Sensitivity is excellent in inherited disorders, and costs have declined over time, especially with multiplexing. Research using genome-wide linkage analysis in families with specific phenotypes led to the identification of MCFD or LMAN1 in combined FV + FVIII deficiency [9] and VKORC1 in Vitamin K-dependent coagulation factor deficiency, type 2 [10]. For clinical diagnostics, there may yet be some distance to cover. Next-generation targeted capture probes against genomic regions already known to be involved in rare heritable bleeding disorders have been evaluated. The Thrombo-Genomics project, whose high-throughput sequencing panel detects variants in 63 genes implicated in bleeding and thrombotic states could, in a recent study on 300 patients, identify $100 \%$ of patients with a previously identified pathogenic variant, $92 \%$ of undiagnosed patients in whom a specific phenotypic diagnosis was suspected, but only $11 \%$ of those without a suspected diagnosis [11]. The remaining $24 \%$ (73/300) cases without a molecular label even after NGS are planned to be submitted to the 100,000 genomes project and many may well prove to be novel disease entities.

As we identify more and more mutations in the heritable bleeding disorders, it has been suggested that a possible cost-effective laboratory workflow could be the use of an upfront NGS panel including 50-100 specific genes with subsequent software interrogation of only the genes relevant to the symptoms and phenotypes of individual patients [8]. This would help in disorders like the HermanskyPudlack syndrome or FXIII deficiency with more than one gene requiring analysis ( 9 and 2 respectively in the examples cited) and also in cases with a phenotypic overlap between disorders, for e.g. in patients with a mild mucocutaneous bleeding phenotype but normal screening coagulogram $[5,8,11]$. In conclusion therefore, it seems safe to predict that diagnostic approaches to the molecular diagnosis of inherited bleeding diatheses are likely to undergo a sea-change over the next decade.

\section{References}

1. Khaniani MS, Ebrahimi A, Daraei S, Derakhshan SM (2016) Genotyping of intron inversions and point mutations in exon 14 of the FVIII gene in Iranian Azeri Turkish families with hemophilia A. Indian J Hemato Blood Transfus. doi:10.1007/s12288016-0699-2

2. Pinto P, Ghosh K, Shetty S (2016) F8 gene mutation profile in Indian hemophilia A patients: identification of 23 novel mutations and factor VIII inhibitor risk association. Mutat Res 786:27-33

3. Toulon P, Eloit Y, Smahi M, Sigaud C, Jambou D, Fischer F, Appert-Flory A (2016) In vitro sensitivity of different activated partial thromboplastin time reagents to mild clotting factor deficiencies. Int J Lab Hematol 38:389-396

4. Jury C, Nagai Y, Tatsumi N (2011) Chapter 1: Collection and handling of blood. In: Bain BJ et al (eds) Dacie and LewisPractical Haematology (11/e). Elsevier, London, pp 1-9

5. Lillicrap D (2013) Molecular testing for disorders of hemostasis. Int J Lab Hematol 35:290-296

6. Fogarty PF, Kessler CM (2013) Hemophilia A and B. In: Kitchens CS, Kessler CM, Konkle BA (eds) Consultative hemostasis and thrombosis, 3rd edn. Elsevier Saunders, Philadelphia, pp 45-59

7. http://www.ncbi.nlm.nih.gov/gene/7450. Accessed 13 September 2016

8. Goodeve AC, Pavlova A, Oldenburg J (2014) Genomics of bleeding disorders. Haemophilia 20(Suppl 4):50-53

9. Zhang B, McGee B, Yamaoka JS, Guglielmone H, Downes KA, Minoldo $S$ et al (2006) Combined deficiency of factor $V$ and factor VIII is due to mutations in either LMAN1 or MCFD2. Blood 107:1903-1907

10. Rost S, Fregin A, Ivaskevicius V, Conzelmann E, Hörtnagel K, Pelz HJ et al (2004) Mutations in VKORC1 cause warfarin resistance and multiple coagulation factor deficiency type 2 . Nature 427:537-541

11. Simeoni I, Stephens JC, Hu F, Deevi SV, Megy K, Bariana TK et al (2016) A high-throughput sequencing test for diagnosing inherited bleeding, thrombotic, and platelet disorders. Blood 127:2791-2803 